\title{
Diversidad de mariposas diurnas (Lepidoptera: Papilionoidea) asociadas con tres hábitats en el corregimiento de Pacurita, municipio de Quibdó, Chocó, Colombia
}

\author{
๑ Víctor Danilo Palacios-Mayoral*, $\odot$ Leison Palacios-Mosquera, ๑Alex Mauricio Jiménez-Ortega \\ Grupo de Investigación en Manejo y Gestión de la Vida Silvestre del Chocó, Facultad de Ciencias Naturales y Exactas, \\ Programa de Biología, Universidad Tecnológica del Chocó, Quibdó, Colombia
}

\begin{abstract}
Resumen
Se determinó la diversidad de mariposas diurnas asociadas a tres hábitats con diferentes grados de conservación en el corregimiento de Pacurita, Quibdó, Chocó, Colombia. Para la captura de mariposas durante 38 días de muestreos entre los meses de julio y diciembre del 2016, se empleó una red entomológica y seis trampas Van SomerenRydon cebadas con pescado en descomposición y frutas fermentadas. Se capturaron 284 individuos de 44 especies pertenecientes a las familias Nymphalidae $(S=38)$ Papilionidae $(S=3)$, Pieridae $(S=1)$ y Riodinidae $(S=2)$. Según el tipo de hábitats, las especies más abundantes fueron Pierella luna lesbia y Heliconius erato venus en el hábitat de bosque conservado; Pierella luna lesbia, Heliconius erato venus y Colobura dirce en el bosque secundario, y Anartia jatrophae, Heliconius erato venus y Anartia amathea en el hábitat de intervención permanente. En los hábitats de bosque conservado y bosque secundario se registró un $62 \%$ de similitud en cuanto a la composición y la estructura de las especies de mariposas, lo cual no ocurrió en el hábitat de intervención permanente. De las 44 especies reportadas en este estudio, 25 constituyen nuevos registros para la localidad de Pacurita, siendo la familia Nymphalidae la predominante. Las especies $P$. luna lesbia, A. jatrophae, H. erato venus, A. demophon muson y $S$. magdalena cleove fueron las mejor representadas, aunque con cambios en su abundancia según el tipo de hábitat. (C) 2018. Acad. Colomb. Cienc. Ex. Fis. Nat.
\end{abstract}

Palabras claves: Biondicadores; Riqueza; Chocó biogeográfico; Conservación; Incidencia de hábitat; Nymphalidae.

The diversity of butterflies (Lepidoptera: Papilionoidea) associated with three habitats in Pacurita, Quibdó municipality, Chocó, Colombia

\begin{abstract}
We determined the diversity of butterflies associated with three habitats with different level of conservation in the village of Pacurita, Quibdó, Chocó, Colombia. For the capture of butterflies during 38 days of sampling between the months of July and December 2016, an entomological network and six Van Someren-Rydon traps baited with decaying fish and fermented fruits were used. A total of 284 individuals of 44 species were captured, from the families Nymphalidae $(\mathrm{S}=38)$, Papilionidae $(\mathrm{S}=3)$, Pieridae $(\mathrm{S}=1)$, and Riodinidae $(\mathrm{S}=2)$. The most abundant species were Pierella luna lesbia and Heliconius erato venus in the preserved forest; Pierella luna lesbia, Heliconius erato venus, and Colobura dirce in the secondary forest, and Anartia jatrophae, Heliconius erato venus, and Anartia amathea in the habitat of permanent intervention. In the preserved and secondary forests, there was a $62 \%$ similarity in the composition and structure of butterfly species, but not so in the permanent intervention habitat. Out of the 44 species reported in this study, 25 constitute new species records for Pacurita locality; the Nymphalidae family was the predominant one. The species $P$. luna lesbia, A. jatrophae, $H$. erato venus, A. demophon muson, and $S$. magdalena cleove were the best represented despite experiencing changes in their abundance according to the type of habitat. (C) 2018. Acad. Colomb. Cienc. Ex. Fis. Nat.
\end{abstract}

Key words: Bioindicators; wealth; Chocó biogeographical region; conservation; incidence over habitats; Nymphalidae.

*Correspondencia:

Víctor Danilo Palacios-Mayoral, danilopalaciosmayoral@gmail.com

Recibido: 18 de enero de 2018, Aceptado: 9 de agosto de 2018, Editor: Elizabeth Castañeda 


\section{Introducción}

Los lepidópteros (mariposas y polillas) comprenden el segundo orden de insectos en cuanto a diversidad, con 157,424 especies descritas (Van Nieukerken, et al., 2011), de las cuales cerca del 11,5\% corresponde a las mariposas diurnas (Papilionoidea). En la región neotropical el número de especies de mariposas reconocidas, uno de los grupos de insectos más estudiados, continúa incrementándose (Gaviria-Ortiz \& Henao-Bañol, 2011; Fratello, et al., 2015; Willmott, et al., 2017) como resultado de las investigaciones en sitios poco estudiados y de la utilización de nuevas técnicas en la delimitación de las especies (Willmott, et al., 2017). Entre los países que conforman la región neotropical, Colombia se destaca por ocupar el segundo lugar después de Perú en términos de riqueza de mariposas, con 3.272 especies, de las cuales solo un 15,3\% aproximadamente se han reportado en el Chocó biogeográfico colombiano (Andrade, et al., 2007).

Las mariposas diurnas son un componente importante de la diversidad de insectos en nuestro país por su gran riqueza y diversidad, la fácil observación y manejo en campo, la estabilidad espacio-temporal, una taxonomía bien documentada, la amplitud de ocupación de hábitats y rangos geográficos, la fidelidad ecológica, la fragilidad frente a perturbaciones mínimas y su corta temporalidad generacional, factores que se han consideradas importantes biondicadores del estado de conservación de los ecosistemas (Kremen, et al., 1993; Andrade, 1998; Fagua, et al., 1999; Valencia, et al., 2005). Además, son organismos sensibles a los cambios de temperatura, el microclima, la humedad y el nivel de luminosidad, aspectos que cambian con la alteración de los ecosistemas (Kremen, et al., 1993). Además de estos atributos, las mariposas también presentan una gran especificidad ecológica frente a sus plantas hospederas y nutricias (Camero, et al., 2007), hecho que ha llevado a diversos investigadores a aseverar que la pérdida de cobertura boscosa afecta la diversidad de las mariposas diurnas (Brown \& Freitas, 2000; Brown \& Freitas, 2002; Huertas \& Ríos, 2006).

En el país se han realizado varios estudios sobre la diversidad de las mariposas diurnas (Prieto \& Constantino, 1996; Fagua, et al., 1999; García, 2008; Vargas-Zapata, et al., 2011; Boom-Urueta, et al., 2013; Marín, et al., 2014), los cuales han acotado diferentes temáticas. Asimismo, investigaciones realizadas en el departamento del Chocó (Ruiz, 2009; González-Valencia, 2012; Salazar-Maya, 2012; Vargas \& Salazar, 2014), han derivado en la documentación de la riqueza en la zona centro, la cual se estima, a la fecha, en aproximadamente 470 especies de mariposas diurnas, es decir, el 14,3\% de las especies reportadas para Colombia (Andrade, et al., 2007).

Son manifiestos los avances logrados en la documentación de la riqueza de mariposas en la región chocoana, especialmente en estudios como el de González-Valencia (2012), quien evaluó la riqueza asociada a un gradiente de perturbación en el corregimiento de Pacurita, zona que, a pesar de ser parte integral del corredor de conservación Tumbes-Chocó-Magdalena (Myers, et al., 2000), presenta problemas de perturbación debidos al desarrollo de actividades humanas (tala selectiva de madera, extracción de metales como oro y platino, etc.). Las mariposas se ven afectadas por dicha situación y constituyen un grupo de insectos que permite evaluar las dinámicas ecológicas de los ecosistemas (Andrade, 1998; Valencia, et al., 2005). En este sentido, el uso del suelo en la zona de estudio, así como su heterogeneidad ambiental y las variaciones en la arquitectura de la vegetación, exige sumar esfuerzos de vigilancia y de documentación de la riqueza de mariposas en hábitats con diferentes estados de conservación. Bajo esta premisa, el objetivo del presente estudio fue determinar la diversidad de mariposas diurnas asociadas a tres hábitats en el corregimiento de Pacurita.

\section{Metodología}

Área de estudio. El trabajo se desarrolló en el corregimiento de Pacurita, el cual está ubicado sobre la margen izquierda del río Cabí, a 5 41' 1.7" N y $76^{\circ} 35$ 49,6" O (municipio de Quibdó) y a $37 \mathrm{~m}$ de elevación; tiene una extensión de $180 \mathrm{Km}^{2}$ y presenta selvas moderadamente alteradas por pequeñas perturbaciones naturales (caída de árboles y ramas) y antrópicas (tala selectiva de maderas finas y minería). Esta zona pertenece a la pluvisilva del Chocó biogeográfico colombiano, localizada en el occidente del país, en el valle del río Atrato, departamento del Chocó. Comprende los municipios de Atrato, Bojayá, Lloró, Istmina, Quibdó, Medio Atrato y Unión Panamericana (Bechara- Escudero \& Jiménez-Ortega, 2009). La zona presenta una temperatura megatermal $\left(25,7\right.$ a $\left.27,9^{\circ} \mathrm{C}\right)$, precipitación de hasta 13.670 $\mathrm{mm}$ y un balance hídrico entre perhúmedo y superhúmedo (248,9 a 385,8) (Poveda, et al., 2004). Desde el punto de vista de la conservación, forma parte integral del corredor de conservación Chocó-Manabí, la zona más importante en términos de biodiversidad de la ecorregión terrestre prioritaria (ETP) de Tumbes-Chocó-Magdalena, reconocida mundialmente como una de las de mayor diversidad biológica, hábitat de muchas especies únicas de flora y fauna (Critical Ecosystem Partnership Funf-CEPF, 2002).

Con base en el nivel de intervención en el área de estudio, se establecieron las siguientes tres zonas de muestreo en el corregimiento de Pacurita (Figura 1): una en un hábitat de bosque conservado, es decir, ubicada en un bosque natural no intervenido; una en un hábitat de bosque secundario que incluía sitios con predominio de crecimiento secundario después de aproximadamente 20 años de haber sido aprovechada para extracción de madera, oro y platino (conocida también como de crecimiento secundario), y una en un hábitat de intervención permanente, caracterizado por presentar parches de bosque con claros, especies de árboles con baja cobertura, rastrojos, áreas de cultivos y árboles frutales: papaya (Carica papaya), limón (Citrus limon), 


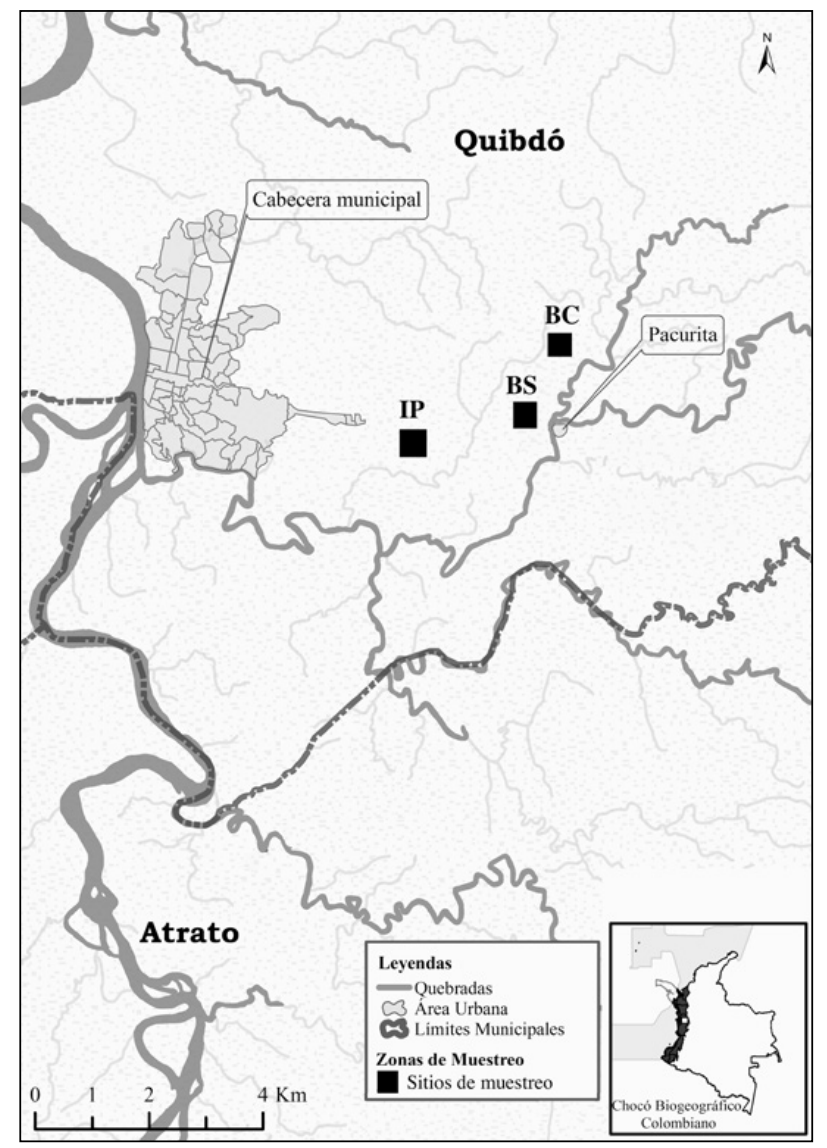

Figura 1. Localización geográfica de tres hábitats en el corregimiento de Pacurita, municipio de Quibdó, Chocó. BC: bosque conservado; BS: bosque secundario; IP: intervención permanente

milpesos (Oenocarpus bátava), guayaba (Psidium guajava) guamo (Inga edulis), paco (Gustavia superba), y yarumo uva (Cecropia peltata) (Quinto-Mosquera, et al., 2013).

Métodos de captura y determinación taxonómica de las mariposas. En cada hábitat se delimitó una parcela de $500 \mathrm{~m}$ de largo por $250 \mathrm{~m}$ de ancho, donde se capturaron las mariposas aplicando una combinación de técnicas de captura según las descritas por Villarreal, et al. (2004) y GonzálezValencia (2012), con algunas variaciones. Se emplearon seis trampas Van Someren-Rydon (VSR) instaladas en línea recta a una distancia de $50 \mathrm{~m}$ una de otra y colgadas en árboles a tres metros del suelo entre las 6:00 y las 18:00 horas durante 38 días. En cada trampa se pusieron cebos con atrayentes como pescado en descomposición y frutas fermentadas: banano (Musa paradisiaca, Linnaeus 1753), borojó (Alibertia patinoi Cuatrecasas 1949), lulo (Solanum quitoense, Lamarck 1794), guayaba (Psidium guajava, Linnaeus 1753) y mango (Mangifera indica, Linnaeus 1753). Las trampas se revisaban cada hora. Además, se empleó una red entomológica durante el mismo periodo de exposición de las trampas según las indicaciones de Villarreal, et al. (2004), con algunas modificaciones, es decir durante ocho (8) horas diarias (cuatro en la mañana y cuatro en la tarde).
Los especímenes capturados con estos métodos se sacrificaron mediante presión digital en el tórax (Neild, 1991) y se preservaron en sobres de papel milano, rotulado previamente con los datos de la captura (método de captura, hora y fecha, tipo de hábitat y recolector), siguiendo los protocolos descritos por Andrade, et al. (2013). Posteriormente fueron tratados en el laboratorio del Grupo de Investigación en Manejo y Gestión de la Vida Silvestre del Chocó, donde se hizo la extensión alar en cada individuo para luego identificarlo.

La determinación taxonómica de los ejemplares se hizo con base en claves y guías ilustradas especializadas (Andrade, 1990; Andrade, et al., 2007; García-Robledo, et al., 2002; Valencia, et al., 2005; D’Abrera, 2006; Warren, et al., 2013), con ayuda de especialistas y mediante el contraste con el material de referencia de la colección entomológica de la Universidad Tecnológica del Chocó.

Análisis estadístico. Se graficaron las curvas de acumulación de especies de cada hábitat estudiado con base en el esfuerzo de muestreo como lo recomiendan Gotelli \& Colwell (2001): al graficar, en el eje X se representa la acumulación de unidades de esfuerzo, por lo que la curva que se genera corresponde a la acumulación de nuevas especies en función del aumento del esfuerzo. Cada gráfica está compuesta de una curva de las especies observadas y curvas a partir de los diferentes estimadores de riqueza, calculados con el programa Estimates 9.1 (Colwell C, 2016), con una aleatorización de 100 iteraciones para crear curvas suavizadas y eliminar posibles errores debidos al orden de las muestras y la heterogeneidad ambiental (Colwell \& Coddington, 1994; Moreno \& Halffter, 2000). Se tuvieron en cuenta los estimadores de riqueza Chao 1 y Jack 1, los cuales usan algoritmos que predicen la diversidad esperada (Moreno, 2001). Debido a la desigualdad de las muestras se hizo una curva de rarefacción para estandarizar los muestreos y así poder hacer comparaciones (Moreno, 2001).

Se evaluó la similitud entre los hábitats mediante un análisis de similitudes (analysis of similarities, ANOSIM) y de agrupamiento de Bray-Curtis, escogido por ser uno de los métodos más ampliamente utilizados y de mayor confiabilidad para la comparación de comunidades (Bloom, 1981).

\section{Resultados}

Se registraron 284 individuos, con un esfuerzo de muestreo total de 3.040 horas/trampas (2.736 horas/trampas Van Someren-Rydon (VSR) y 304 horas/red -hombre), para un éxito de captura de 0,2840 individuos/horas/trampas y red entomológica (Tabla 1). El total de los registros comprendió cuatro familias, 11 subfamilias y 44 especies, de las cuales Pierella luna lesbia $(\mathrm{n}=56)$, Anartia jatrophae $(\mathrm{n}=36)$, Heliconius erato venus $(\mathrm{n}=31)$, Archaeoprepona demophon muson $(\mathrm{n}=14)$, Stalachtis magdalena cleove $(\mathrm{n}=13)$, Colobura dirce $(\mathrm{n}=11)$, Pierella helvina hymettia $(\mathrm{n}=11)$ y Heliconius hecale melicerta $(\mathrm{n}=10)$ fueron las más abundantes. La familia con mayor número de especies fue 
Tabla 1. Esfuerzo y éxito de captura en tres hábitats en el corregimiento de Pacurita, Quibdó, Chocó, Colombia. BC: bosque conservado, BS: bosque secundario; IP: intervención permanente

\begin{tabular}{cccc}
\hline Hábitats & $\begin{array}{c}\text { Esfuerzo de muestreo } \\
\text { (horas-trampa y horas-red/hombre) }\end{array}$ & $\begin{array}{c}\text { Individuos capturados } \\
\text { (n) }\end{array}$ & $\begin{array}{c}\text { Éxito de captura } \\
\text { (individuos/horas-trampa y red) }\end{array}$ \\
\hline BC & 1200 & 88 & 0,0733 \\
BS & 1040 & 119 & 0,1144 \\
IP & 800 & 77 & 0,0963 \\
Total & $\mathbf{3 0 4 0}$ & $\mathbf{2 8 4}$ & $\mathbf{0 , 2 8 4 0}$ \\
\hline
\end{tabular}

la Nymphalidae, con 38 especies $(n=264)$ equivalentes al $93 \%$ de las capturas; las familias Papilionidae, Riodinidae y Pieridae tuvieron poca representatividad, con tan solo tres, dos y una especie, respectivamente. Asimismo, las subfamilias Satyrinae, con 11 especies, y Nymphalinae y Heliconiinae, con seis especies cada una, fueron las más abundantes y predominantes en los tres hábitats (Tabla 2, Figura 2).

Representatividad de los muestreos de mariposas en los tres hábitats estudiados. Las curvas de acumulación de especies para los tres hábitats no mostraron una asíntota en el número de especies capturadas, lo que sugiere que se requieren más muestreos para documentar una mayor cantidad de muestras y aumentar el número de especies de mariposas reportadas para los tres hábitats muestreados en el corregimiento de Pacurita, zona centro de la selva pluvial tropical del Chocó (Figura 3). Al estandarizar el esfuerzo de muestreo en los hábitats a partir de la curva de rarefacción con base en un número de 10 unidades de muestreos comparables, se estableció que el hábitat de bosque conservado arrojó el menor número de especies, mientras que los hábitats de bosque secundario e intervención permanente presentaron la mayor riqueza de especies (Figura 4).

Con esfuerzos de muestreo diferentes, se encontró que el hábitat de bosque secundario presentó la mayor abundancia y riqueza de especies $(n=119, \mathrm{~s}=28)$ pertenecientes a $10 \mathrm{sub}$ familias y tres familias); las espe-cies predominantes fueron $P$. luna lesbia, H. erato venus. En el hábitat de bosque conservado se capturaron 88 individuos agrupados en 24 especies de mariposas, 10 subfamilias y dos familias. Las especies predominantes en este hábitat fueron P. luna lesbia, H. erato venus, $A$. demophon muson, en tanto que en el hábitat de intervención permanente se capturaron 77 individuos agrupados en 16 especies, nueve subfamilias y tres familias. Anartia jatrophae, $H$. erato venus y $A$. amathea fueron las especies más predominantes en este hábitat (Tabla 2).

Según el índice de Bray-Curtis, se presentó una similitud del $62 \%$ entre los hábitats de bosque conservado y bosque secundario, a diferencia del hábitat de intervención permanente que fue el hábitat más disímil, con tan solo un $18 \%$ de especies compartidas (Figura 5). El hábitat de bosque conservado presentó ocho especies exclusivas, seguido por el hábitat de intervención permanente, con siete especies, y, por último, el hábitat de bosque secundario con seis especies (Tabla1). De las 44 especies capturadas en este estudio, solo tres se encontraron simultáneamente en los tres hábitats: Adelpha boeotia boeotia (C. Felder \& R. Felder, 1867), C. dirce (Linnaeus, 1758) y H. erato venus (Staudinger, 1882) (Tabla 2).

\section{Discusión}

Los datos obtenidos en esta investigación permiten inferir que con el esfuerzo de muestreo y los dos métodos empleados, se logró la documentación de 44 especies de mariposas diurnas, que corresponden al $40,7 \%$ de las especies reportadas por González-Valencia (2012) para tres hábitats con diferentes grados de perturbación en el corregimiento de Pacurita.

La mayor diversidad de la familia Nymphalidae en los tres hábitats estudiados concuerda con lo reportado por otros autores (Prieto \& Constantino, 1996; Fagua, et al., 1999; Campos \& Andrade, 2007; Ruiz, 2009; Orozco, et al., 2009; González-Valencia, 2012; Salazar-Maya, 2012; Boom-Urueta, et al., 2013; Vargas \& Salazar, 2014). Esta mayor representatividad puede estar relacionada con el hecho de que dicha familia presenta el mayor número de subfamilias, géneros y especies de mariposas diurnas (Campos \& Andrade, 2007). Sumado a esto, la riqueza de Nymphalidae también se puede atribuir a la alta disponibilidad de plantas hospederas de las familias Arecaceae, Maranthaceae y Heliconiaceae en la región del Chocó (Palacios \& Constantino, 2006), apreciación que compartimos, al ser esta familia una de las de mayor presencia en los hábitats donde se desarrollaron nuestros muestreos. Además, Nymphalidae posee cerca del $31 \%$ de especies de mariposas diurnas del Neotrópico y el $30 \%$ de las especies reportadas para Colombia (Lamas, 2000).

La baja representatividad de especies de mariposas en las otras familias, en particular la Papilionidae, puede deberse a la escasez de plantas hospederas de las familias Aristolochiaceae (Prince-Chacón, et al., 2011), a lo cual se sumarían los posibles sesgos en las capturas debido a la coloración opaca que presentan la mayoría de especies de la familia Riodinidae, las cuales se ocultan en el follaje del sotobosque y en el borde de bosque, y a que algunas de sus especies habitan en las partes altas del dosel y pasan desapercibidas (García-Robledo, et al., 2002; CamposSalazar \& Andrade, 2007). 
Tabla 2. Composición taxonómica de mariposas diurnas (Papilionoidea) en tres hábitats en el corregimiento de Pacurita, Quibdó, Chocó, Colombia. BC: bosque conservado; BS; bosque secundario; IP: intervención permanente; RD: red entomológica; TVSR: trampas Van Someren-Rydon; n: número de individuos por especies

\begin{tabular}{|c|c|c|c|c|c|c|c|}
\hline \multirow[t]{2}{*}{ Familia/Subfamilia/Especie } & \multicolumn{3}{|c|}{ Hábitats } & \multicolumn{2}{|c|}{ Métodos de captura } & \multicolumn{2}{|c|}{ Abundancia } \\
\hline & $\mathbf{B C}$ & BS & IP & RED & TVSR & $\mathbf{n}$ & $\%$ \\
\hline \multicolumn{8}{|l|}{ Nymphalidae $(S=38 ; n=264)$} \\
\hline \multicolumn{8}{|l|}{ Biblidinae $(\mathrm{S}=\mathbf{3} ; \mathrm{n}=\mathbf{5})$} \\
\hline Catanophele orites (Stichel, 1899) & 1 & 2 & 0 & 2 & 1 & 3 & 0,01 \\
\hline Hamadryas sp & 1 & 0 & 0 & 1 & 0 & 1 & 0,003 \\
\hline Hamadryas feronia farinulenta (Fruhstorfer, 1916) & 0 & 0 & 1 & 1 & 0 & 1 & 0,003 \\
\hline \multicolumn{8}{|l|}{ Charaxinae $(S=2 ; N=15)$} \\
\hline Archaeoprepona demophon muson (Fruhstorfer, 1905) & 9 & 5 & 0 & 9 & 5 & 14 & 0,049 \\
\hline Memphis pseudiphis(Staudinger, 1887) & 0 & 0 & 1 & 1 & 0 & 1 & 0,003 \\
\hline \multicolumn{8}{|l|}{ Danainae $(\mathrm{S}=5 ; \mathrm{N}=\mathbf{2 3})$} \\
\hline Aeria eurimedia ssp. & 3 & 3 & 0 & 6 & 0 & 6 & 0,021 \\
\hline Hypoleria lavinia libera (Godman \& Salvin, 1879) & 0 & 6 & 0 & 6 & 0 & 6 & 0,021 \\
\hline Lycorea cleobaea atergatis(Doubleday,1847) & 0 & 1 & 1 & 1 & 1 & 2 & 0,01 \\
\hline Napeogenes stella opacella (Kaye, 1918) & 1 & 6 & 0 & 7 & 0 & 7 & 0,024 \\
\hline Oleria amalda amaldina (Haensch, 1909) & 1 & 1 & 0 & 2 & 0 & 2 & 0,007 \\
\hline \multicolumn{8}{|l|}{ Heliconiinae $(S=6 ; N=50)$} \\
\hline Dryas iulia iulia (Fabricius, 1775) & 0 & 1 & 1 & 2 & 0 & 2 & 0,007 \\
\hline Heliconius cydno ssp. & 2 & 0 & 0 & 2 & 0 & 2 & 0,007 \\
\hline Heliconius erato venus (Staudinger, 1882) & 10 & 9 & 12 & 27 & 4 & 31 & 0,109 \\
\hline Heliconius hecale melicerta (H. Bates, 1866) & 0 & 5 & 5 & 9 & 1 & 10 & 0,035 \\
\hline Heliconius hecalesia ssp & 0 & 2 & 1 & 1 & 2 & 3 & 0,01 \\
\hline Heliconius $s p$ & 0 & 2 & 0 & 1 & 1 & 2 & 0,007 \\
\hline \multicolumn{8}{|l|}{ Limenitidinae $(S=2 ; N=5)$} \\
\hline Adelpha sp & 0 & 1 & 1 & 0 & 2 & 2 & 0,007 \\
\hline Adelpha boeotia boeotia (C. Felder \& R. Felder, 1867) & 1 & 1 & 1 & 1 & 2 & 3 & 0,01 \\
\hline \multicolumn{8}{|l|}{ Morphinae $(\mathrm{S}=3 ; \mathrm{N}=9)$} \\
\hline Anthirrhea tomasia (Butler, 1875) & 1 & 0 & 0 & 0 & 1 & 1 & 0,003 \\
\hline Morpho cypris lelargei (Oberthür, 1921) & 1 & 0 & 0 & 0 & 1 & 1 & 0,003 \\
\hline Morpho helenor macrophthalmus (Fruhstorfer, 1913) & 7 & 0 & 0 & 3 & 4 & 7 & 0,024 \\
\hline \multicolumn{8}{|l|}{ Nymphalinae $(S=6 ; N=61)$} \\
\hline Anartia amathea (Linnaeus, 1758) & 0 & 0 & 9 & 9 & 0 & 9 & 0,031 \\
\hline Anartia jatrophae (Linnaeus, 1763) & 0 & 0 & 36 & 35 & 1 & 36 & 0,126 \\
\hline Colobura dirce (Linnaeus, 1758) & 3 & 6 & 2 & 7 & 4 & 11 & 0,038 \\
\hline Historis acheronta acheronta(Fabricius, 1775) & 1 & 1 & 0 & 2 & 0 & 2 & 0,007 \\
\hline Historis odius dious (Lamas, 1995) & 0 & 1 & 0 & 0 & 1 & 1 & 0,003 \\
\hline Junonia genoveva(Cramer,1768) & 0 & 0 & 2 & 2 & 0 & 2 & 0,007 \\
\hline \multicolumn{8}{|l|}{ Satyrinae $(S=11 ; N=96)$} \\
\hline Caligo eurilochus ssp & 3 & 0 & 0 & 1 & 2 & 3 & 0,01 \\
\hline Caligo idomeneus ssp. & 2 & 0 & 0 & 1 & 1 & 2 & 0,007 \\
\hline Selenophanes josephus excisus(Rothschild, 1916) & 2 & 1 & 0 & 0 & 3 & 3 & 0,01 \\
\hline Cithaerias pireta (Stoll, 1780) & 2 & 2 & 0 & 4 & 0 & 4 & 0,014 \\
\hline Dulcedo polita (Hewitson, 1869) & 0 & 2 & 0 & 2 & 0 & 2 & 0,007 \\
\hline Eryphanis automedon (Cramer, 1775) & 0 & 1 & 0 & 1 & 0 & 1 & 0,003 \\
\hline Opsiphanes cassina ssp. & 1 & 6 & 0 & 5 & 2 & 7 & 0,024 \\
\hline Pierella helvina hymettia (Staudinger, [1886]) & 4 & 7 & 0 & 10 & 1 & 11 & 0,038 \\
\hline Pierella luna lesbia (Staudinger, 1887) & 25 & 31 & 0 & 46 & 10 & 56 & 0,197 \\
\hline Taygetis laches ssp. & 0 & 0 & 1 & 1 & 0 & 1 & 0,003 \\
\hline Tigridia acesta columbina(Neustetter, 1929) & 5 & 1 & 0 & 1 & 5 & 6 & 0,021 \\
\hline Papilionidae $(S=3 ; n=4)$ & & & & & & & \\
\hline Papilioninae $(S=3 ; n=4)$ & & & & & & & \\
\hline Heraclides thoas nealces (Rothschild \& Jordan, 1906) & 0 & 2 & 0 & 2 & 0 & 2 & 0,007 \\
\hline Parides eurimedes emilius (Constantino, 1999) & 0 & 1 & 0 & 1 & 0 & 1 & 0,003 \\
\hline Protesilaus glaucolaus glaucolaus (H. Bates, 1864) & 0 & 0 & 1 & 1 & 0 & 1 & 0,003 \\
\hline Pieridae $(S=1 ; n=2)$ & & & & & & & \\
\hline Coliadidinae $(S=1 ; n=2)$ & & & & & & & \\
\hline Phoebis sp & 0 & 0 & 2 & 2 & 0 & 2 & 0,007 \\
\hline Riodinidae $(S=2 ; n=14)$ & & & & & & & \\
\hline Riodininae $(S=2 ; n=14)$ & & & & & & & \\
\hline Necyria $s p$ & 1 & 0 & 0 & 0 & 1 & 1 & 0,003 \\
\hline Stalachtis magdalena cleove (Staudinger, 1888) & 1 & 12 & 0 & 13 & 0 & 13 & 0,045 \\
\hline Total de individuos & 88 & 119 & 77 & 228 & 56 & 284 & \\
\hline Total de especies & 24 & 28 & 16 & & & & \\
\hline Especies exclusivas & 8 & 6 & 7 & & & 21 & \\
\hline Especies compartidas & 3 & 3 & 3 & & & & \\
\hline
\end{tabular}




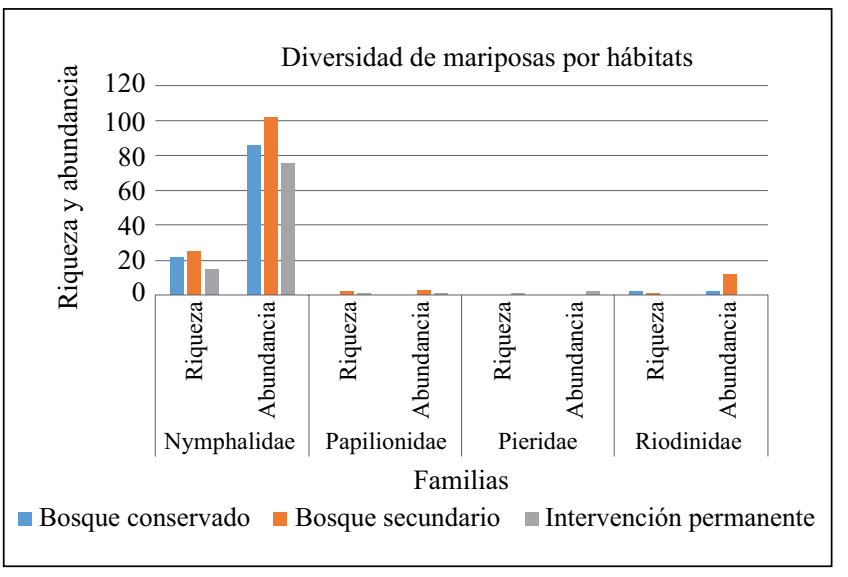

Figura 2. Riqueza y abundancia de mariposas diurnas (Papilionoidea) en tres hábitats con diferente grado de intervención en el corregimiento de Pacurita, Quibdó, Chocó, Colombia

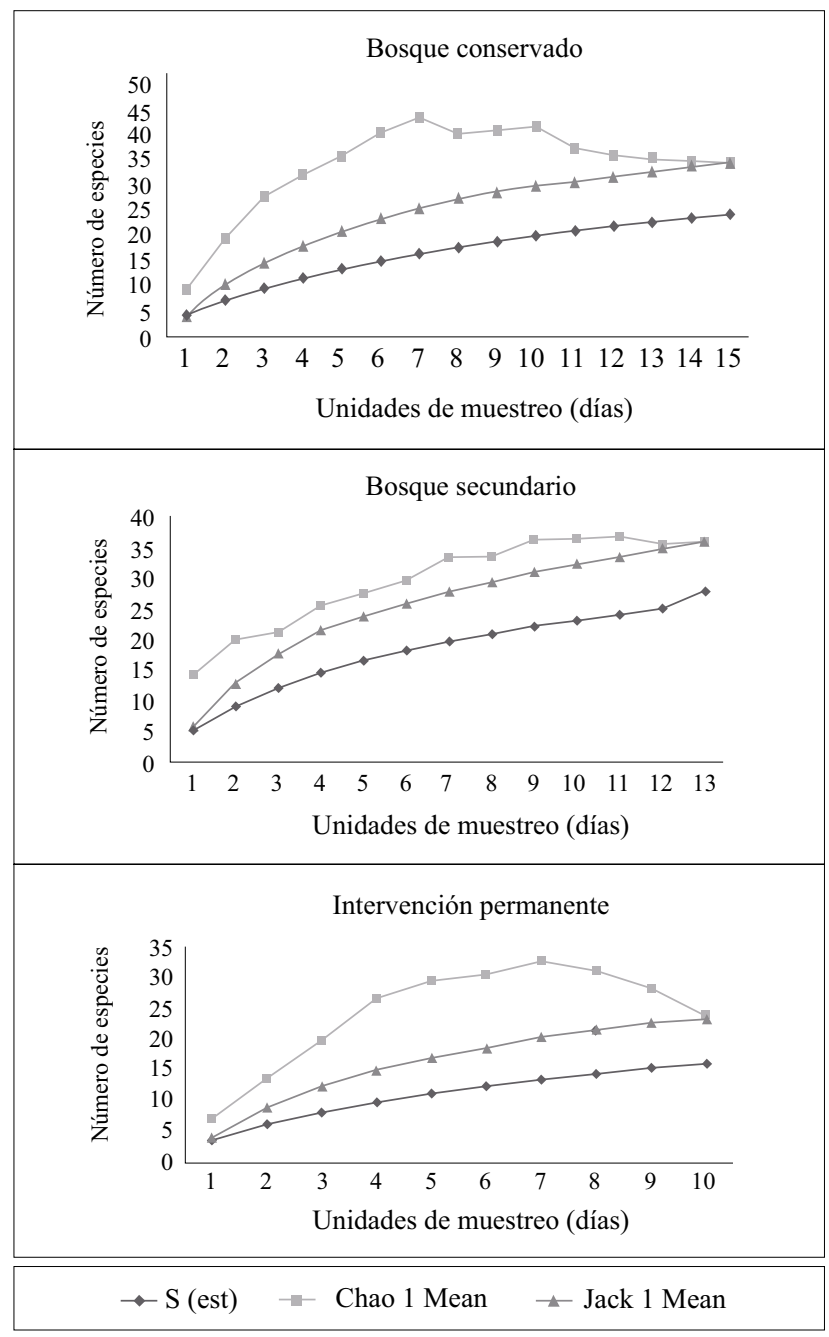

Figura 3. Curvas de acumulación de especies para los tres hábitats de muestreo en bosque conservado (BC), bosque secundario (BS) e intervención permanente (IP) en el corregimiento de Pacurita, municipio de Quibdó
Aunque los estimadores de riqueza indican la necesidad de sumar más unidades de muestreo, la mayor representatividad de las especies $P$. luna lesbia, A. jatrophae, $H$. erato venus, $C$. dirce y $P$. helvina hymettia (Tabla 1), coincide con lo encontrado por González-Valencia (2012) y SalazarMaya (2012), quienes las reportan como las especies de mayor abundancia.

En este estudio se reportan 25 especies adicionales a las 108 registradas por González-Valencia (2012), lo cual incrementa a 132 el número de especies de mariposas diurnas en la zona, con una coincidencia de apenas $17,6 \%$ de las especies encontradas por esta misma autora (A. boeotia boeotia (C. Felder y R. Felder, 1867), Adelpha sp., Aeria eurimedia ssp., Caligo eurilochus ssp., Caligo idomeneus ssp., (Cramer, 1775), Dryas iulia iulia (Fabricius, 1775), Eryphanis automedon (Cramer, 1775), Hamadryas feronia farinulenta (Fruhstorfer, 1916), Hamadryas sp., Heliconius cydno ssp, Heliconius hecale melicerta (H. Bates, 1866), Heliconius hecalesia ssp., Heliconius sp., Hypoleria lavinia libera (Godman y Salvin, 1879), Lycorea cleobaea atergatis (Doubleday,1847), Memphis pseudiphis (Staudinger, 1887), Napeogenes stella opacella (Kaye, 1918), Necyria sp.,

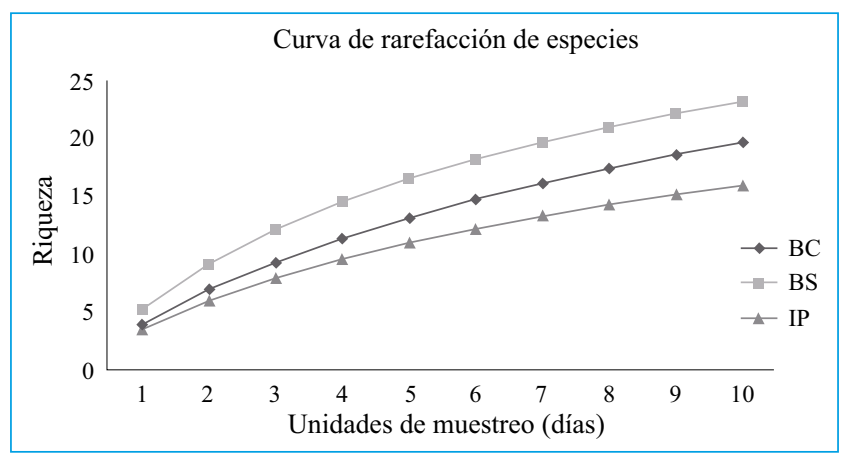

Figura 4. Curva de rarefacción de especies para los tres hábitats de muestreo en bosque conservado (BC), bosque secundario (BS) e intervención permanente (IP) en el corregimiento de Pacurita, municipio de Quibdó

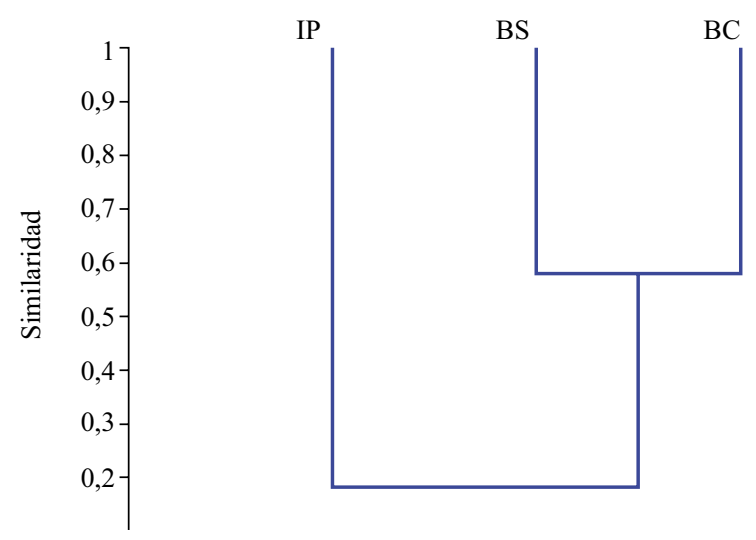

Figura 5. Análisis de similitud en los hábitats de bosque conservado (BC); bosque secundario (BS), e intervención permanente (IP) 
Oleria amalda amaldina (Haensch, 1909), Opsiphanes cassina ssp., Phoebis sp., Protesilaus glaucolaus glaucolaus (H. Bates, 1864), Selenophanes josephus excisus (Rothschild, 1916), Taygetis laches ssp y Tigridia acesta columbina (Neustetter, 1929)). Se resalta el hecho de que en estas dos investigaciones los hábitats de bosque conservado y bosque secundario presentaron la mayor similitud.

La abundancia de la especie Morpho helenor macrophthalmus en el hábitat de bosque conservado puede relacionarse con su vuelo a la altura del dosel de bosques $\mathrm{y}$ bordes de bosques poco intervenidos (Andrade, et al., 2007). El predominio de la especie P. luna lesbia en los hábitats de bosque conservado y bosque secundario, se puede atribuir a su pertenencia a la tribu Haeterini (Satyrinae: Nymphalidae), que presenta especies que vuelan a ras del suelo y a baja altura en caminos sombreados, selvas y bosques tropicales, y se les considera indicadores ecológicos ideales (Constantino, 1995).

La abundancia de las especies $A$. jatrophae y $A$. amathea en el hábitat de intervención permanente, se asocia a que pueden colonizar varios hábitats, por lo cual no se las considera buenas indicadoras del estado de conservación de los bosques (Campos-Salazar, et al., 2011); estas, además, se encuentran en áreas transformadas y perturbadas por el uso del suelo para pastoreo y la presencia de espacios abiertos como bordes de bosque (Vargas-Zapata, et al., 2011). Además, son especies calificadas como indicadoras de hábitats en intervención permanente (Árias \& Huertas, 2001; Valencia, et al., 2005).

La presencia de $C$. dirce en los tres hábitats posiblemente se relaciona con su vuelo asociado a variedad de hábitats: bosques secundarios, claros de bosque, bordes de quebrada y bordes de bosque (Valencia, et al., 2005, Instituto Nacional de Biodiversidad-INBio Web, 2008). Asimismo, la presencia de $A$. boeotia boeotia se asocia con la amplia distribución neotropical del género Adelpha, cuyas especies frecuentan variedad de hábitats como bosques primarios, bosques húmedos, hábitats no perturbados $\mathrm{y}$ antropogénicos, por lo que se las considera indicadoras de la calidad de los ecosistemas, siendo útiles en estudios de conservación y diversidad (Domínguez-Puerta, 2016).

El hallazgo de $H$. erato venus y su abundancia en los tres hábitats estudiados sugiere que la especie es tolerante a cambios en la arquitectura de la vegetación derivados de las condiciones ambientales. Vargas-Zapata, et al. (2012) señalan que los periodos de mayor precipitación y de cambios constantes en la temperatura favorecen la floración de las plantas y proporciona una mayor oferta alimenticia a las mariposas, lo cual incide en el establecimiento de especies del género Heliconius. A pesar de que los miembros de este género presentan alta especificidad ecológica, frecuentan áreas abiertas y bosques secundarios (Hay-Roe, 2008).

En las curvas de acumulación se evidenció la falta de registros de nuevas especies (Figura 3), lo cual coincide con lo señalado por González-Valencia (2012) en el sentido de que las curvas de acumulación de especies no alcanzaron la asíntota para tres zonas en un gradiente de perturbación en el corregimiento de Pacurita (Quibdó), selva pluvial central del Chocó, Colombia.

\section{Conclusiones}

Las 44 especies reportadas en este estudio representan un porcentaje bajo de la riqueza estimada, según lo sugerido por los estimadores de riqueza, aunque se adicionaron 25 nuevos registros de especies para la localidad de Pacurita. Sin embargo, dada la heterogeneidad ambiental de esta zona, su riqueza de mariposas está aún subestimada.

Las especies $P$. luna lesbia, A. jatrophae, $H$. erato venus, $A$. demophon muson y S. magdalena cleove fueron las mejor representadas a pesar de que su abundancia reflejó cambios según el tipo de hábitat. Por otra parte, la familia Nymphalidae se reconoció como la más predominante, registrando una gran diversidad y el mayor número especies.

El aumento de zonas abiertas puede estar afectando la riqueza de mariposas, en tanto que las zonas más conservadas mantendrían la riqueza de especies en términos de su composición y estructura, lo que contrasta con la mayor similitud entre los hábitats de bosque conservado y bosque secundario.

\section{Agradecimientos}

Los autores expresamos los más sinceros agradecimientos al equipo de colaboradores del Grupo de investigación en Manejo y Gestión de la Vida Silvestre del Chocó por su apoyo logístico para el desarrollo de esta investigación; a la comunidad de Pacurita por su hospitalidad al permitirnos realizar el levantamiento de información en campo; al curador de la colección entomológica de la Universidad Tecnológica del Chocó, Leyser Rengifo Murillo, por sus aportes en el proceso curatorial de los ejemplares, y a Efraín Reinel Henao Bañol por su colaboración en la identificación de los especímenes.

\section{Contribución de los autores}

Víctor Danilo Palacios-Mayoral: revisión de la literatura, toma de datos en campo, preservación y montaje de mariposas en el laboratorio, identificación taxonómica, análisis estadísticos, redacción del manuscrito, proceso editorial. Leison Palacios-Mosquera: toma de datos en campo, revisión de la literatura, análisis de datos, redacción del manuscrito, proceso editorial. Alex Mauricio JiménezOrtega: análisis de datos, redacción del manuscrito

\section{Conflicto de intereses}

Los autores declaran que no existe conflicto de intereses.

\section{Referencias}

Andrade, M.G. (1990). Clave para las familias y subfamilias de Lepidóptera: Rhopalocerca de Colombia. Caldasia. 16: 539-550.

Andrade, M.G. (1998). Utilización de mariposas como biondicadoras del tipo de hábitats y su biodiversidad en Colombia. Rev. Acad.Colomb. Cienc. 22 (84): 407-421. 
Andrade, M.G., Campos-Salazar, L.R., González-Montaña L.A., Pulido-B, H.W. (2007). Santa María mariposas alas y color. Serie de Guías de Campo del Instituto de Ciencias Naturales No. 2. Instituto de Ciencias Naturales, Universidad Nacional de Colombia, Bogotá D. C., Colombia. p. 248.

Andrade, M.G., Henao-Bañol, E.R., Triviño, P. (2013). Técnicas y procesamiento para la recolección, preservación y montaje de Mariposas en estudios de biodiversidad y conservación. (Lepidóptera: Hesperioidea - Papilionoidea). Rev. Acad. Colomb. Cienc. 37 (144): 311-325.

Arias, J.J. \& Huertas-H.B.C. (2001). Mariposas diurnas de la Serranía de los Churumbelos, Cauca. Distribución altitudinal y diversidad de especies (Lepidóptera: Rhopalocera: Papilionoidea). Revista Colombiana de Entomología. 27 (3-4): 106-109.

Bechara-Escudero, M. \& Jiménez-Ortega, A.M. (2009). Dieta entomofágica en una población de Rinella marina en la selva pluvial central, Chocó, Colombia. Investigación, Biodiversidad y Desarrollo. 28 (2): 216-21.

Bloom, S.A. (1981) Similarity indices in community studies: potential pitfalls. Marine Ecology Progress Series. 5: 125-128.

Boom-Urueta, C., Seña-Ramos, L., Vargas-Zapata, M., Martínez-Hernández, N. (2013). Mariposas Hesperioidea y Papilionoidea (Insecta: Lepidóptera) en un fragmento de bosque seco tropical, Atlántico, Colombia. Bol.Cient. Mus. Hist. Nat. 17 (1):149-167.

Brown, K.J. \& Freitas, L.A.V. (2000). Atlantic forest butterflies: Indicators for Landscape Conservation. Biotropica. 32 (4b): 934-956.

Brown, K.J. \& Freitas, L.A.V. (2002). Butterfly communities of the urban forest fragments in Campinas, Sao Paulo, Brazil: Structure, instability and environmental correlates and conservation. Journal of Insect Conservation. 6: 217-231.

Camero, E. \& Calderón, A.M. (2007). Comunidad de mariposas diurnas (Lepidóptera: Rhopalocera) en un gradiente altitudinal del cañón del Rio Combeima-Tolima, Colombia. Acta biol. Colomb. 12 (2): 295-110.

Campos-Salazar, L.N \& Andrade-C, M.G. (2007). Lepidópteros (Mariposas). En: Estudio de la caracterización biológica y ecológica integral, Fase I: diagnóstico, evaluación y planificación del proceso de recuperación, protección y conservación del bosque natural del Agüil en el municipio de Aguachica, y Fase II: formulación del área protectora del Agüil, municipio de Aguachica-Cesar. Rangel-Ch., editor. p. 103-116.

Campos-Salazar, L.R., Gómez-Bulla, J., Andrade-C, M.G. (2011). Mariposas (Lepidóptera: Hesperioidea-Papilionoidea) de las áreas circundantes a las ciénagas del Departamento de Córdoba, Colombia. Rev. Acad. Colomb. Cienc. 35 (134): 45-60.

Critical Ecosystem Partnership Funf-CEPF. (2002). Corredor Chocó-Manabí. Ecorregión (Hostpots) de Biodiversidad del Chocó- Darién-Ecuador Occidental. Hoja de Datos del CEPF. Washington, DC.

Constantino, LM. (1995). Revisión de la tribu Haeterini HerrichSchaffer, 1864 en Colombia (Lepidoptera: Nymphalidae, Satyrinae). SHILAP Revta. /lepid. 23 (89): 49-76.

Colwell, R.K. \& Coddington, J.A. (1994). Estimating terrestrial biodiversity through extrapolation. Philosophical Transactions of the Royal Society (Series B). 345: 101-118.
Colwell, R.K. (2016). EstimateS: Biodiversity estimation. Current Version Estimates 9.1.0. Fecha de consulta: entre Junio y diciembre de 2017. Disponible en: viceroy.eeb.uconn.edu/ estimates

D’Abrera, B. (2006). World Butterflies. Hill House. Publishers. Melbourne/London. p. 272.

Domínguez-Puerta, K.L. (2016). Sistemática y distribución del género Adelpha Hübner, [1819] (Lepidoptera: Nymphalidae: Limenitidinae) en Colombia. (MSc). Universidad Nacional. p. 278.

Fagua, G., Amarillo-S, A.R., Andrade-C, M.G. (1999). Mariposas (Lepidoptera) como biondicadores del grado de intervención en la cuenca del Río Pato (Caquetá). P. 285-315. En: Amat-G, G., Andrade-C, M.G., Fernández, F. (editores). Insectos de Colombia. Volumen II. Academia Colombiana de Ciencias Exactas, Físicas y Naturales. Colección Jorge Álvarez Lleras N¹3. Bogotá. p. 433.

Fratello, S.A., Nakahara, S., Brévignon, C.R., Harvey, D.J. (2015). Two new species of Euptychia Hübner, 1818 (Lepidoptera: Nymphalidae: Satyrinae) from the Guiana Shield, with notes on E. marceli Brévignon, 2005 and E. rufocincta Weymer, 1911. The Journal of the Lepidopterists' Society. 69 (4): 293-306.

García, J.A. (2008). Diagnóstico ambiental del relleno sanitario praderas del Magdalena, Girardot (Cundinamarca) empleando mariposas (Lepidóptera: Papilionoidea y Hesperioidea) como indicador de tipo de calidad de hábitat. Revista Tumbaga. 3: 92-108.

García-Robledo, C.A., Constantino, L.M., Heredia, M.D., Katan, J. (2002). Mariposas comunes de la cordillera Central de Colombia. Wildlife Conservation Society, Programa Colombia. Cali, Colombia. p. 130.

Gaviria-Ortiz, F.G. \& Henao-Bañol, E.R. (2011). Diversidad de mariposas diurnas (Hesperioidea-Papilionoidea) del Parque Natural Regional el Vínculo (Buga-Valle del Cauca). Boletín Científico Museo Historia Natural. 15: 115-133.

González-Valencia, E.L. (2012). Estructura y composición de ensamblaje de mariposas Diurnas (Rhopalocera) en un gradiente de perturbación en la selva pluvial central del Chocó, Colombia. Tesis de Pregrado. Quibdó-Colombia, Universidad Tecnológica del Chocó "Diego Luis Córdoba". Facultad de Ciencias Básicas. p. 66.

Gotelli, N.J. \& Colwell, R.K. (2001). Quantifying biodiversity: Procedures and pitfalls in the measurement and comparison of species richness. Ecology Letters. 4: 379-391.

Hay-Roe, M.M. (2008). Races of Heliconius erato (Nymphalidae: Heliconiinae) found on different sides of the Andes show wing size differences. McGuire Center for the Lepidoptera and Biodiversity. Florida Entomologist. 91 (4): 711-712.

Huertas, B. \& Ríos, J.C. (2006). Estudio de las mariposas diurnas de la serranía de los Yariguíes y su conservación (Lepidóptera: Papilionoidea) (en) Huertas, B.C \& Donegan, T.M. (Editores.). Proyecto Yaré: Investigación y evaluación de las especies amenazadas de la serranía de los Yariguíes, Santander, Colombia. BP Conservation Programme. Colombian EBA Project Report Series. 7: 44.55.

Instituto Nacional de Biodiversidad - INBio. (2008). Servicios de información. Fecha de consulta: octubre de 2008. Disponible en: http://www.inbio.ac.cr/otus/servicios.html

Kremen, C, Colwell, R.K., Erwin, T.L., Murphy, D.D., Noss, R.F. Sanjkayan, M.A. (1993). Terrestrial arthropod assemblages: Their use in Conservation Planning. Conservation Biology. 7 (4): 796-808. 
Lamas, G. (2000). Estado actual del conocimiento de la sistemática de los lepidópteros, con especial referencia a la región neotropical. Monografías Tercer Milenio. 1: 253-260.

Marín, M.A., Álvarez, C.F., Giraldo, C.E., Pyrcz, T.W., Uribe, S.I. Vila, R. (2014). Mariposas en un bosque de niebla andino periurbano en el valle de Aburrá, Colombia. Revista Mexicana de Biodiversidad. 85: 200-208.

Myers, N., Mittermeier, R.A., Mittermeier, C.G., Fonseca, G.A.B., Ken D.A.J. (2000). Biodiversity Hotspots for Conservation Priorities. Nature. 403 (24): 853-859.

Moreno, C.E. \& Halffter, G. (2000). Assessing the completeness of bat biodiversity inventories using species accumulation curves. Journal of Applied Ecology. 37: 149-158.

Moreno CE. 2001. Métodos para medir la biodiversidad. M \& TManuales y Tesis SEA. vol.1. Zaragoza, p. 84.

Neild, A.F.E. (1991). The butterflies of Venezuela. Part 1. (Nymphalidae. I. Limetidinae, Apaturinae, Charaxinae). A comprehensive guide to identification of adult Nymphalidae, Papilionidae and Pieridae. Meridian Publications, London, p. 1-144.

Orozco, S., Muriel, S.B., Palacios, J. (2009). Diversidad de lepidópteros diurnos en un área de bosque seco tropical del occidente Antioqueño. Actual Biol. 31 (90): 31-41.

Palacios, M. \& Constantino, L.M. (2006). Diversidad de lepidópteros Rhopalocera en un gradiente altitudinal en la reserva natural el pangan, Nariño, Colombia. Bol.Mus. Ent.Univ. 10: $258-278$.

Poveda, I.C., Rojas, C., Rudas, A. Rangel-CH, J.O. (2004) El Chocó biogeográfico: ambiente físico. In: Rangel-Ch., JO., Diversidad Biótica IV, El Chocó Biogeográfico/ Costa Pacífica Universidad Nacional de Colombia. Bogotá D.C. $1-22$.

Prieto-M, A.D. \& Constantino, L.M. (1996). Abundancia, distribución y diversidad de mariposas (Lep. Rhopalocera) en el río Tatabro, Buenaventura (Valle-Colombia). Bol. Mus. Ent.Univ. Valle. 4 (2):11-18.

Prince-Chacón S., Vargas-Zapata M.V., Salazar J. MartínezHernández N.J. (2011). Mariposas Papilionoidea y Hesperioidea (Insecta: Lepidoptera) en dos fragmentos de bosque seco tropical en corrales de San Luis, Atlántico, Colombia. Boletín de la Sociedad Entomológica Aragonesa (S.E.A.). 48: 243-252.

Quinto-Mosquera, J., Moreno-Amud, L.A., MosqueraMosquera, S., Mantilla-Meluk, H. Jiménez-Ortega, A.M. (2013). Cambios en el ensamblaje de murciélagos filostómidos producto de la perturbación en el distrito Alto Atrato-San Juan, Chocó, Colombia. Investigación, Biodiversidad y Desarrollo. 32 (2): 121-32.

Ruiz, R.C.Y. (2009). Caracterización taxonómica y ecológica de la comunidad de Lepidópteros Rhopalocera en la Cuenca del río Cabí, Chocó, Colombia. Tesis de postgrado. Universidad Tecnológica del Chocó "Diego Luís Córdoba", Facultad Ciencias Básicas. p. 48.
Salazar-Maya, M.C. (2012). Diversidad de lepidópteros Rhopalocera en un bosque de la selva pluvial central (sector La Platina, Chocó-Colombia) y descripción del ciclo de vida de una especie presente en la zona de estudio. Tesis de Pregrado. Quibdó-Chocó, Universidad Tecnológica del Chocó "Diego Luís Córdoba", Facultad Ciencias Básicas. p. 95.

Van Nieukerken, E.J., Kaila, L., Kitching, I.J., Kristensen, N.P., Lees, D.C., Minet, J., Mitter, C., Mutanen, M., Regier, J.C., Simonsen, T.J., Wahlberg, N., Yen, S.-H., Zahiri, R., Adamski, D., Baixeras, J., Bartsch, D., Bengtsson, B.Å., Brown, J.W., Bucheli, S.R., Davis, D.R., De Prins, J., De Prins, W., Epstein, M.E., Gentili-Poole, P., Gielis, C., Hättenschwiler, P., Hausmann, A., Holloway, J.D., Kallies, A., Karsholt, O., Kawahara, A.Y., Koster, J.C., Kozlov, M.V., Lafontaine, J.D., Lamas, G., et al. (2011). Order Lepidoptera Linnaeus, 1758. In Z.-Q. Zhang (editor.), Animal biodiversity: An outline of higher-level classification and survey of taxonomic richness. Zootaxa. 3148: $212-221$.

Valencia, M., Gil, Z.N. Constantino, L.M. (2005). Mariposas diurnas de la zona central cafetera colombiana. Guía de campo, Chinchiná (Colombia). Cenicafe. p. 244.

Vargas-CH, J.I., Salazar-E, C.A. (2014). Biodiversidad y mariposas en una región del alto Chocó, San José del Palmar, Colombia. Bol. Cient. Mus. Hist. Nat. U. de Caldas. 18 (1): 259-284.

Vargas-Zapata, M.A., Martínez-Hernández, N.J., GutiérrezMoreno, L.C., Prince-Chacón, S., Herrera-Colon, L.F. (2011). Riqueza y abundancia de Hesperioidea y Papilionoidea (Lepidoptera) en la Reserva Natural Las Delicias, Santa Marta, Magdalena, Colombia. Acta Biol. Colomb. 16 (1): 43-60.

Vargas-Zapatas, M.A, Prince-Chacón, S., Martínez-Hernández, N.J. (2012). Estructura poblacional de Heliconius erato hydara Hewitson, 1867 (Lepidoptera: Nymphalidae) en la reserva campesina la montaña (RCM), departamento del Atlántico, Colombia. Boletín de la Sociedad Entomológica Aragonesa (S.E.A.). 51: 273-281.

Villarreal, H., Álvarez, H.M., Córdoba, S., Escobar, F., Fagua, G., Gast, F., Mendoza, H., Ospina, M., Umaña, A.M. (2004). Insectos. En: Manual de métodos para el desarrollo de inventarios de biodiversidad. Bogotá (Colombia): Instituto de Investigación de Recursos Biológicos Alexander von Humboldt. p. 151-183.

Warren, A.D., Davis K.J., Stangeland, E.M., Pelham, J.P., Grishinn, V. (2013). Illustrated List of American Butterflies. [20- VII- 2013].

Willmott, K. R., Simon, M. J., Ortiz-Acevedo, E., Hall, J.P. (2017). First record of the enigmatic tribe Anaeomorphini (Lepidoptera, Nymphalidae, Charaxinae) outside of the Amazon basin: A new species of Anaeomorpha Rothschild, 1894, from the Chocó region of western Ecuador. Publicación Insecta Mundi. 0548: 1-10. 